\title{
State Estimation of The Underwater Moving Target Based on Multi-Sensor Information Fusion
}

\author{
Zheng Tang, Chao Sun, Zong-wei Liu \\ Institute of Acoustical Engineering \\ Northwestern Polytechnical University \\ Xi'an, China \\ e-mail: adamtangzheng@nwpu.edu.cn
}

\author{
Di Meng \\ Northwestern Polytechnical University \\ Xi'an, China
}

\begin{abstract}
The Kalman filter has been widely used in state estimation of moving targets. Furthermore, the well-known conventional Kalman filter requires an accurate system model and exact prior information. However, the nonlinearity, dynamic and randomicity in the underwater environment result in uncertainty and incontinuity of the observation information and unknown bias of the system model, which may seriously degrade the performance of the Kalman filter or even cause the filter to diverge. Therefore, a novel filtering algorithm based on multi-sensor information fusion estimation theory and dynamic bayesian network inference is presented, which is based on the idea of fusing firstly and then filtering. Firstly, the multi-sensor system fuses the sub-systems measurement information to obtain more accurate initial measurement information and covariance information, and then smooth missing data and fuzzy data by fusing the the obtained system state predictive information and all the measurement information of the sub-systems to obtain the accurate state estimation of underwater moving target. Finally, the simulation results show that the proposed method can efficiently estimate underwater target state without prior noise information, and can evidently improve the state estimation precision of underwater moving target by adjusting the weight factor despite noise-related.
\end{abstract}

Keywords-State estimation; Underwater moving target; Multi-sensor information fusion

\section{INTRODUCTION}

The state estimation of the underwater moving target is more difficult than one of the immobile target, because the nonlinear, dynamic and random in underwater evironment result in uncertainty and incontinuity of the observation information and unknown bias of the system model. Many existing methods need accurate prior information and only apply to the linear system [1-4], so can not be used directly.

Several researchers improved the existing methods. Ref.[5] proposes an adaptive two-stage extended Kalman filter using an adaptive fading EKF, and applies it to the inertial navigation system-global positioning system loosely coupled system with an unknown fault bias for estimating the unknown bias effectively although the information about the random bias was unknown. Ref.[6] proposes the sensor information fusion Kalman filter based on the introduced statistics of mathematical expectation of the spectral norm of a normalized innovation matrix. The approach allows for simultaneous test of the mathematical expectation and the variance of innovation sequence in real time and does not require a priori information on values of the change in its statistical characteristics under faults. Ref.[7] extend the cubature Kalman filter to deal with nonlinear state-space models of the continuous-discrete kind, and use the ItôTaylor expansion of order 1.5 to transform the process equation, modeled in the form of stochastic ordinary differential equations, into a set of stochastic difference equations. Ref.[8] proposes the relaying Kalman filter algorithm which introduce the equations of updating sensor probability, and reconstruct the innovation equation. Furthermore, the extension of the proposed method can be applied in nonlinear dynamic system. Although these methods can estimate the target motion state in nonlinear system, they cannot accurately estimate the motion state of underwater target while observations are uncertainty and incontinuity.

This paper proposed a novel filtering algorithm based on the dynamic optimization weighted covariance fusion and the forwards-backwards inference algorithm of dynamic bayesian network. While the noise information is unknown and relevant, the dynamic optimization weighted covariance fusion method can obtain the higher accurate measurements by adjusting fusion weight with changing of sensor signals, and offer the accurate prior information next filtering. Moreover, the filter theory based on dynamic bayesian network inference is the theory of sequentially estimating the underlying state of a system using measurements obtained over time, which combines the evidences before a certain time slice and the evidences after this time slice by the forwards-backwards inference algorithm of dynamic bayesian network to smooth the missing data and the fuzzy data, and accurately estimate the underwater target motion state. Finally, the simulation results show that the proposed method can evidently improve the state estimation precision of underwater moving target.

\section{State Estimation Model Based On The Multi- SENSOR INFORMATION FUSION}

The multi-sensor information fusion is a process that deal with the association, correlation, and combination of the information collected from various sensors. In order to accurately estimate the underwater target state, the dynamic optimization weighted covariance fusion method is chosen to 
reduce the noise interference caused by sensor measurement through adjusting fusion weight with changing of sensor error covariance. Moreover, the forwards-backwards inference algorithm based on dynamic bayesian network combines the previous fusion result with current sensory information from various sensor platforms to infer and smooth the underlying state of the underwater target. With reality, the state estimation model of underwater moving target based on multi-sensor information fusion is established as Fig. 1.

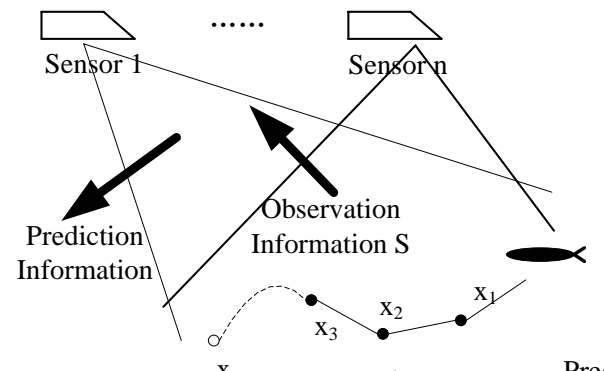

(a)

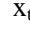

Track

Predicting

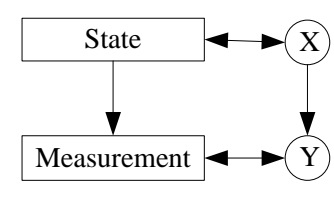

(b)

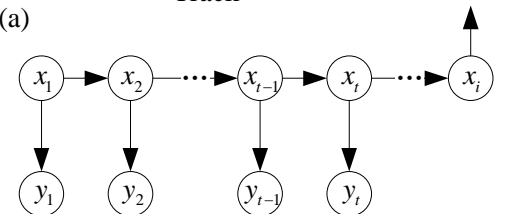

(c)
Fig. 1 The state estimation model of the underwater moving target based on multi-sensor information fusion

The real target track is expressed as $x_{1}, \cdots, x_{t}$ shown in Fig.1(a). But due to the system error and the measurement error, the optimization state estimation only can be obtained by filtering and smooth. In other words, the implied real state sequence $\mathbf{X}$ can be obtained by inferring and filtering from the observation sequence, as shown in Fig.1(b) and Fig.1(c).

\section{Dynamic Optimizaiton WeIghted Covariance FUSION}

Consider an ad-hoc sensor network of $m$ sensors at positions $s_{1}, s_{2}, \ldots, s_{m}$. Assume that there is only one target of interest whose state equation is driven by process noise. At any time $t$, the state of the target evolves according to

$$
\mathbf{X}(t)=\mathbf{F} \mathbf{X}(t-1)+\mathbf{w}(t)
$$

where $\mathbf{F}$ is the state transition matrix, $\mathbf{w}(t)$ is zeromean white Gaussian noise vector of variance $\mathbf{Q}$ caused by disturbances and modelling errors. The measurement model of sensor $i$ is given by

$$
\mathbf{Y}_{i}(t)=\mathbf{H}_{i} \mathbf{X}(t)+\mathbf{n}_{i}(t), \quad i=1,2, \cdots, m
$$

where $\mathbf{Y}_{i}(t)$ is the observation vector of sensor $i, \mathbf{H}_{i}$ is the observation matrix, $\mathbf{n}_{i}(t)$ is the zero-mean additive white Gaussian noise of variance $\mathbf{R}$. The measurements error of each sensor at time $t$ is expressed as

$\mathbf{e}_{i}(t)=\mathbf{H}_{i}(t) \hat{\mathbf{X}}(t \mid t-1)-\mathbf{Y}_{i}(t), \quad i=1,2, \cdots, m$

As above, the weighted measurement error is given as

$$
\tilde{\mathbf{e}}(t)=\left[w_{1}(t) \mathbf{e}_{1}(t), w_{2}(t) \mathbf{e}_{2}(t), \cdots, w_{m}(t) \mathbf{e}_{m}(t)\right]^{T}
$$

According to the least squares criterion, the sum of the square measurement error is obtained as

$$
\tilde{\mathbf{e}}^{T}(t) \tilde{\mathbf{e}}(t)=\sum_{i=1}^{m}\left(w_{i}(t) \mathbf{e}_{i}(t)\right)^{2}, \quad i=1,2, \cdots, m
$$

In terms of lagrange criterion and constraints of $\sum_{i=1}^{m} w_{i}=1$, the minimum of equation (5) is

$$
w_{i}(t)=\frac{\mathbf{e}_{i}^{-1}(t)}{\sum_{j=1}^{m} \mathbf{e}_{j}^{-1}(t)} \quad i=1,2, \cdots, m
$$

Then, the optimization fusion estimates of the state and the covariance can be respectively expressed as:

$$
\begin{aligned}
\hat{\mathbf{X}}^{*} & =\sum_{i=1}^{m} w_{i}(t) \mathbf{Y}_{i}(t)=\sum_{i=1}^{m} \frac{\mathbf{e}_{i}^{-1}(t) \mathbf{Y}_{i}(t)}{\sum_{j=1}^{m} \mathbf{e}_{j}^{-1}(t)} \\
\mathbf{P}^{*} & =\sum_{i=1}^{m} w_{i}(t) \mathbf{P}(t)=\sum_{i=1}^{m} \frac{\mathbf{e}_{i}^{-1}(t) \mathbf{P}(t)}{\sum_{j=1}^{m} \mathbf{e}_{j}^{-1}(t)}
\end{aligned}
$$

From equations (7) and (8), several properties of the information weight can be concluded, shown in following

1) The information weight on itself in (6) is equal to the variance of the information. The larger the information weight is, the higher the estimation accuracy is.

2) The sum of the weight of all the measurement informaiton of the state is equal to the information weight of the optimal fusion estimation on itself in (7).

3) When the information weight is zero, the measurement information is useless to the state. When the information weight is infinite, the measurement information is completely accurate state information. 


\section{FORWARDS-BACKWARDS INFERENCE ALGORITHM BASED ON DYNAMIC BAYESIAN NERWORK}

In process of dynamic bayesian network inference, we attempt to construct the posterior probability density function of the state given all measurements. All available information is used to form such a probability density function. So this probability density function will represents complete solution.

It is assumed that $\mathbf{X}(t)$ is the state sequence, which has $n$ states. $\mathbf{Y}(t)$ is the measurement, which is represented as $y_{1: m}=y_{1}, y_{2}, \cdots, y_{m}$. The $t$ is defined $t=1,2, \cdots, T$ to represent the $t$ time slices on the networks. The state transition matrix is $\mathbf{F}=\left(f_{i j}\right)_{n \times n}$, which is always given based on system design.

In the process of deduction, we will use two important hypotheses of the classical HMM model:

Assumption 1: State at the $(t+1)$ th time slice only depends on the state at the th time slice, independent from other states.

Assumption 2: The observation probability only depends on the state at the th time slice, independent from other states.

Then, the estimation of $\mathbf{X}(t)$ based on all available measurements at time $t$ (marked as $\mathbf{Y}(1: t)$ ) can be obtained by constructing the posterior probability density function $P(\mathbf{X}(t), \mathbf{Y}(1: t))$, which can be obtained recursively by the forwards-backwards algorithm (FBA) in two stages, namely prediction stage and update stage. Suppose that the required probability density function $P(\mathbf{X}(t) \mid \mathbf{Y}(1: t))$ at time step $t-1$ is available. According to the forwards inference algorithm, $\alpha_{t}(j)=P\left(X_{t}=j \mid y_{1,1: m}, y_{2,1: m}, \cdots, y_{t, 1: m}\right) \quad$ is computed recursively as follows,

$$
\begin{aligned}
\alpha_{t}(j)= & P\left(X_{t}=j \mid y_{1,1: m}, y_{2,1: m}, \cdots, y_{t, 1: m}\right) \\
= & \prod_{k=1}^{m} P\left(Y_{t k}=y_{t k} \mid X_{t}=j\right) \sum_{i=1}^{n} a_{i j} \\
& \times P\left(X_{t-1}=i \mid y_{1,1: m}, \cdots, y_{t-1,1: m}\right) \\
= & \prod_{k=1}^{m} P\left(Y_{t k}=y_{t k} \mid X_{t}=j\right) \sum_{i=1}^{n} a_{i j} \alpha_{t-1}(i)
\end{aligned}
$$

By equation (9), we can obtain the optimization estimates of the state and the covariance, which is represented as:

$$
\hat{\mathbf{X}}(t \mid t-1)=\mathbf{F} \hat{\mathbf{X}}(t-1 \mid t-1)
$$

$$
\mathbf{P}(t \mid t-1)=\mathbf{F P}(t-1 \mid t-1) \mathbf{F}^{T}
$$

where $\hat{\mathbf{X}}(t-1 \mid t-1)$ and $\mathbf{P}(t-1 \mid t-1)$ are replaced by $\hat{\mathbf{X}}^{*}$ and $\mathbf{P}^{*}$, respectively.

According to the obtained estimates of the state and the covariance at time $t$, we can predict the target state at time $t+1$ by the backwards inference algorithm, such as $\beta_{t}(i)=P\left(y_{t+1,1: m}, y_{t+2,1: m}, \cdots, y_{T, 1: m} \mid X_{t}=i\right)$, which can be computed recursively as follows,

$$
\begin{aligned}
\beta_{t}(i)= & \sum_{j=1}^{n} P\left(y_{t+2,1: m}, \cdots, y_{T, 1: m}, X_{t+1}=j, y_{t+1,1: m} \mid X_{t}=i\right) \\
= & \sum_{j=1}^{n} P\left(y_{t+2,1: m}, \cdots, y_{T, 1: m} \mid X_{t+1}=j\right) \\
& \times P\left(y_{t+1,1: m} \mid X_{t+1}=j\right) P\left(X_{t+1}=j \mid X_{t}=i\right) \\
= & \sum_{j=1}^{n} \beta_{t+1}(j) \prod_{k=1}^{m} P\left(y_{t+1, k} \mid X_{t+1}=j\right) a_{i j}
\end{aligned}
$$

By equation (12), we can obtain the predictive value of the state and the covariance, which is described as:

$$
\begin{gathered}
\hat{\mathbf{X}}(t+1 \mid t)=\mathbf{F} \hat{\mathbf{X}}(t \mid t) \\
\mathbf{P}(t+1 \mid t)=\mathbf{F P}(t \mid t) \mathbf{F}^{T}
\end{gathered}
$$

The update stage can be described by the following equations:

$$
\begin{gathered}
\mathbf{e}(t)=\mathbf{Y}(t)-\mathbf{H} \hat{\mathbf{X}}(t \mid t-1) \\
\mathbf{S}(t)=\mathbf{H P}(t \mid t-1) \mathbf{H}^{T} \\
\mathbf{K}(t)=\mathbf{P}(t \mid t-1) \mathbf{H}^{T} \mathbf{S}^{-1}(t)
\end{gathered}
$$

where $\mathbf{Y}(t)$ is the system observation at time $t, \mathbf{S}(t)$ is innovation covariance, $\mathbf{K}(t)$ is gain.

The update state estimates and the update covariance are respectively expressed as

$$
\hat{\mathbf{X}}(t \mid t)=\hat{\mathbf{X}}(t \mid t-1)+\mathbf{K}(t) \mathbf{e}(t)
$$




$$
\mathbf{P}(t \mid t)=(\mathbf{I}-\mathbf{K}(t) \mathbf{H}) \mathbf{P}(t \mid t-1)
$$

To sum up, the measurement is used to modify the predicted prior from the previous time step to obtain the state probability density function. Simultaneously, the current measurement and estimation are used to predict the future state probability density function. By recursively computing, the system can complete state estimation and prediction at any time.

\section{The Simulation EXAMPLES}

In order to verify the proposed model and algorithm can evidently improve the precision of state estimates, we assume that the initial state of underwater moving target is set as:

$$
\mathbf{X}=\left[\begin{array}{llll}
10 & 10 & 20 & 0
\end{array}\right]^{T}
$$

and system state transition matrix, observations matrix, system state noise vector and observations noise vector are respectively presented as:

$$
\begin{aligned}
& \mathbf{F}=\left[\begin{array}{llll}
1 & 0 & 1 & 0 \\
0 & 1 & 0 & 1 \\
0 & 0 & 1 & 1 \\
0 & 0 & 0 & 1
\end{array}\right], \mathbf{H}=\left[\begin{array}{llll}
1 & 0 & 0 & 0 \\
0 & 1 & 0 & 0
\end{array}\right] \\
& \mathbf{Q}=\left[\begin{array}{cc}
0.1 & 0 \\
0 & 0.1
\end{array}\right], \mathbf{R}=\left[\begin{array}{cccc}
20 & 0 & 0 & 0 \\
0 & 20 & 0 & 0 \\
0 & 0 & 20 & 0 \\
0 & 0 & 0 & 20
\end{array}\right]
\end{aligned}
$$

Then, state estimation of underwater target is implemented by Kalman filtering and proposed method with 40 sampling points. The simulation results can be shown as Fig. 2.

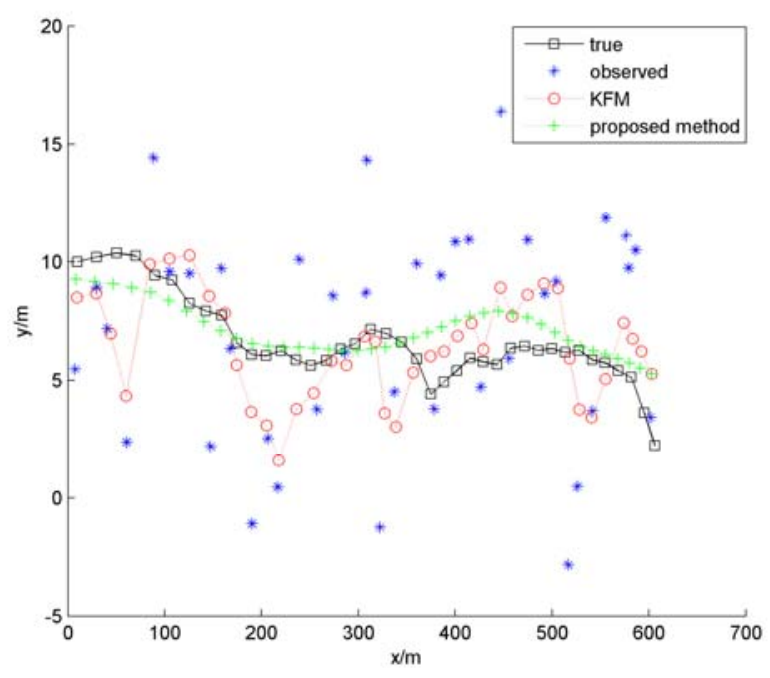

Fig. 2 The simulation result of state estimation
Compared simulation results in Fig. 2, we can see that the state estimates obtained by proposed method is evidently better than by Kalman filtering, and efficiently improve the precision of the target. The proposed method firstly reduces the system noise influence by the weighted covariance fusion, which is based on the distributed fusion theory. And then, it uses the forwards-backwards data repairing algorithm for repairing data, which was to use the combined evidences before a certain time slice and the evidences after this time slice to estimate the missing data or fuzzy data on this time slice. Moreover, the proposed method not only can be used in estimation of the highly non-linear time series, but also in estimation of the discrete non-linear.

\section{CONCLUSION}

The filtering algorithm based on multi-sensors information fusion is extend based on the existing filtering algorithms. It not only can smooth the missing data and the fuzzy data by fusing the predictive state information and all the measurement information of the sub-systems, but also can efficiently restrain the noise interference by adjusting fusion weight with changing of sensor signals. Moreover, the proposed method can be used in a nonlinear stochastic system with an unknown random bias. Finally, the experimental results show that the proposed method can efficiently estimate underwater target state without the noise prior information, and can evidently improve the state estimates precision of the underwater moving target by adjusting the weight factor despite noise-related.

\section{REFERENCES}

[1] Y.F. Hu, J.C. Sun, "Study of Underwater Passive Motion Target Analysis in Revise Extend Kalman Filter,” ACTA ACUSTICA, vol.27, no.5, pp.449-454, Sep. 2002.

[2] J. Gao, W.S. Yan, D.M. Xu, F.B. Zhang, "Unscented Kalman Filter for Passive Underwater Target Tracking,” Fire Control and Command Control, vol.31, no.12, pp.26-29, Dec. 2006.

[3] J.H. He, X.G. Wu, H. Yang, “Torpedo Speed Estimation Based on Nonlinear Kalman Filter,” Journal of Projectiles, Rockets, Missiles and Guidance, vol.28, no.5, pp.271-280, Oct. 2008.

[4] S. Gao, Q. Pan, Q.K. Xiao, "Maneuvering Target Tracking Based on Extended Kalman Filter,” Fire Control and Command Control, vol.34, no.9, pp.14-17, Sep. 2009.

[5] H.K. Kwang, G.L. Jang, G.P. Chan, “Adaptive Two-stage Extended Kalman Filter for A Fault-tolerant INS-GPS Loosely Coupled System,” IEEE Trans. On Aerospace and Electronics Systems vol.45, no.1, pp.125-137, Jan. 2009.

[6] O. Ali, H. Chingiz, H. Ulviyye, "Fault Detection in Sensor Electronics and Communications, vol.63, no.3, pp.762-768, Jun. 2009.

[7] I. Arasaratnam, S. Haykin, R.H. Thomas, "Cubature Kalman Filtering for Continuous Discrete Systems: Theory and Simulations,” IEEE Trans. On Signal Processing, vol.58, no.10, pp.4977-4993, Oct. 2010.

[8] Z.G. Liu, J.K. Wang, W. Qu, "Relaying Kalman Filters for Sensor Networks," International Conference on Computer Design and Appliations, vol.2, pp.618-621, Jun. 2010. 